\title{
A New General Decay Rate of Wave Equation with Memory-Type Boundary Control
}

\author{
Sheng Fan \\ Department of Economic Mathematics, Southwestern University of Finance and Economics, Chengdu 611130, China \\ Correspondence should be addressed to Sheng Fan; fansheng@swufe.edu.cn
}

Received 7 January 2021; Revised 16 January 2021; Accepted 23 January 2021; Published 13 February 2021

Academic Editor: Sundarapandian Vaidyanathan

Copyright (C) 2021 Sheng Fan. This is an open access article distributed under the Creative Commons Attribution License, which permits unrestricted use, distribution, and reproduction in any medium, provided the original work is properly cited.

Of interest is a wave equation with memory-type boundary oscillations, in which the forced oscillations of the rod is given by a memory term at the boundary. We establish a new general decay rate to the system. And it possesses the character of damped oscillations and tends to a finite value for a large time. By assuming the resolvent kernel that is more general than those in previous papers, we establish a more general energy decay result. Hence the result improves earlier results in the literature.

\section{Introduction}

It is well-known that if we add a damping to a system, the amplitude of the oscillations can be reduced very fast. The memory term can be as a damping (viscoelastic damping) which is weaker than frictional damping. For viscoelastic materials, Boltzmann theory gives us that the stress-strain viscoelastic law depending on a relaxation measure, see Prüss [1] and Eden et al. [2]. Based on the Boltzmann principle, the viscoelastic stress-strain relations can be generally given by a convolution term, which can be regarded as a lower order perturbation and can also be regarded as a kind of memory effect, for instance, $g^{*} u$. And we call the function $g(t)$ memory kernel. One can find a detail derivation on some systems with memory in [3].

To motivate our work, we start with some known results on wave equation with memory-type oscillations. The general wave equation with viscoelastic term in the internal feedback

$$
u_{t t}-\Delta u+\int_{0}^{t} g(t-s) \Delta u(s) \mathrm{d} s=\mathscr{F}(u) .
$$

Messaoudi and Messaoudi $[4,5]$ studied $\mathscr{F}(u)=0$ and $\mathscr{F}(u)=|u|^{\rho} u$, by introducing the assumption $g^{\prime}(t) \leq-\xi(t) g(t)$, and obtained the energy decays exponentially (polynomially) as $g$ decays exponentially (polynomially), respectively.

Lasiecka et al. [6] considered the general assumption on $g: g^{\prime}(t) \leq-H(g(t))$ to establish general decay of energy. Here $H$, which was introduced by Alabau-Boussouira and Cannarsa [7], is strictly convex and increasing function. Cavalcanti et al. [8, 9], Lasiecka and Wang [10], Mustafa and Messaoudi [11], and Xiao and Liang [12] also used this 
assumption to obtain some general decay rates of corresponding models. In recent papers [13-15], the authors investigated three classes of viscoelastic wave equation as in $[4,5]$ and established optimal and explicit decay results of energy by adopting the assumption on $g$ : $g^{\prime}(t) \leq-\xi(t) H(g(t))$.

In this paper, we considered the following wave equation with boundary oscillations of memory type:

$$
\left\{\begin{array}{l}
u_{t t}-\Delta u=0, \quad \text { in } \Omega \times \mathbb{R}^{+}, \\
u=0, \quad \text { on } \Gamma_{0} \times \mathbb{R}^{+}, \\
u+\int_{0}^{t} g(t-s) \frac{\partial u}{\partial \nu}(s) \mathrm{d} s=0, \quad \text { on } \Gamma_{1} \times \mathbb{R}^{+}, \\
u(x, 0)=u_{0}(x), \\
u_{t}(x, 0)=u_{1}(x), \quad x \in \Omega,
\end{array}\right.
$$

where $\Omega \subset \mathbb{R}^{n}(n \geq 1)$ is a bounded domain with smooth boundary $\Gamma, \Gamma=\Gamma_{0} \cup \Gamma_{1}$, and $\Gamma_{0}$ and $\Gamma_{1}$ are closed and disjoint with measure $\left(\Gamma_{0}\right)>0$. $v$ is the unit outward normal to $\Gamma$.

For wave equation with memory-type boundary oscillations, it can be regarded as a wave equation with viscoelastic damping at the boundary. Santos [16] considered a one-dimensional wave equation with memory conditions at the boundary, respectively. He proved that the energy of solutions decays exponentially (polynomially) as $k$ and $k^{\prime}$ decay exponentially (polynomially). Here $k$ is the resolvent kernel of $\left(-g^{\prime} / g(0)\right)$. Santos et al. [17] extended the results in [16] to an n-dimensional wave equation of Kirchhoff type with memory-type boundary. They proved the global existence of solutions and obtained that the energy of solution decays uniformly with the same rate of decay $k$ under the same conditions on $k$ and $k^{\prime}$, which improves the results in [18] by Park et al. Santos and Junior [19] obtained a similar result for plate equation with memory-type boundary. We also mention the work of Cavalcanti et al. [20], where the authors showed the global existence and the uniform decay of solutions to a semilinear wave equation with memorytype boundary condition and a nonlinear boundary source. Messaoudi and Soufyane [21] considered a general assumption on $k^{\prime}: k^{\prime \prime} \geq-\xi(t) k^{\prime}(t)$ and established a general decay result. $\mathrm{Wu}$ [22] used this assumption to study a wave Kirchhoff-type wave equation with a boundary control of memory type. For nonlinear wave equations with memorytype boundary condition, we refer to Cavalcanti and Guesmia [23], Feng [24], Feng et al. [25-27], Muñoz Rivera and Andrade [28], and Zhang [29].

Concerning the system (2), Mustafa [30], by assuming the function $k: k^{\prime \prime}(t) \geq H\left(-k^{\prime}(t)\right)$, where $k$ is the resolvent kernel of $\left(-g^{\prime} / g(0)\right)$, established a general decay of solutions of the form

$$
E(t) \leq k_{3} H_{1}^{-1}\left(k_{1} t+k_{2}\right), \quad \forall t \geq 0 .
$$

Here

$$
\begin{aligned}
& H_{1}(t)=\int_{t}^{1} \frac{1}{s H_{0}^{\prime}\left(\varepsilon_{0} s\right)} \mathrm{d} s, \\
& H_{0}(t)=H(D(t)),
\end{aligned}
$$

and $D$ is a positive $C^{1}$ function with $D(0)=0$, and $H_{0}$ is strictly increasing and strictly convex $C^{2}$ function on $(0, r]$. In particular, for $H(t)=t^{p}$, i.e., $k^{\prime \prime} \geq c\left(-k^{\prime}\right)^{p}$, the author proved the energy decay holds for $1 \leq p<(3 / 2)$. Whether can the range be extended to a more larger range? In this paper, we give a positive answer to study problem (2) and extend the result to get a more general decay rate. In particular, we obtain that the energy result holds for $H(t)=t^{p}$ with the full admissible range $1 \leq p<2$. More exactly, by assuming the relaxation function $k$ with minimal conditions on $L^{1}(0, \infty)$, i.e., $k^{\prime \prime}(t) \geq \eta(t) H\left(-k^{\prime}(t)\right)$, where $H$ is linear or strictly increasing and strictly convex functions of class $C^{2}\left(\mathbb{R}^{+}\right)$, we establish an optimal explicit and general energy decay result. In particular, the energy result holds for $H(t)=$ $t^{p}$ with the range $p \in[1,2)$ instead of $p \in[1,(3 / 2))$ in [30]. Hence our results extend and improve the stability results in [30] and also in [16-18, 21]. We mainly adopt the idea of $[14,15,31]$ and some properties of convex function developed in $[7,32]$.

The remaining of the paper is organized as follows: in Section 2, we propose some preliminaries. In Section 3, main results are given. Section 4 is devoted to proving the general decay result.

\section{Preliminaries}

Taking the derivative of (2) with respect to $t$, we shall see that

$$
\frac{\partial u}{\partial \nu}=-\frac{1}{g(0)}\left[u_{t}+g_{2}^{\prime} * \frac{\partial u}{\partial \nu}\right]
$$

We denote the resolvent kernel of $\left(-g^{\prime} / g(0)\right)$ by $k$ satisfying for $t \geq 0$ :

$$
k(t)+\frac{1}{g(0)}\left(g^{\prime *} k\right)(t)=-\frac{1}{g(0)} g^{\prime}(t) .
$$

Using Volterra's inverse operator and taking $\alpha=(1 / g(0))$, we have

$$
\frac{\partial u}{\partial v}=-\alpha\left[u_{t}+k_{2} * u_{t}\right]
$$

Assume $u_{0}=0$ on $\Gamma_{1}$ in this paper, we get

$$
\frac{\partial u}{\partial \nu}=-\alpha\left[u_{t}+k(0)+k * u\right], \quad \text { on } \Gamma_{1} \times \mathbb{R}^{+} .
$$
of (2).

In the following, we use boundary conditions (8) instead

As in [30], we consider the following assumption: 
(A1) There exists a fixed point $x_{0} \in \mathbb{R}^{2}$ and some constant $\delta_{0}>0$ such that for $m(x)=x-x_{0}$,

$$
\begin{aligned}
& \Gamma_{0}=\{x \in \Gamma: m(x) \cdot v(x) \leq 0\}, \\
& \Gamma_{1}=\left\{x \in \Gamma: m(x) \cdot v(x) \geq \delta_{0}\right\} .
\end{aligned}
$$

For the kernel $k$, we assume

(A2) $k: \mathbb{R}^{+} \longrightarrow \mathbb{R}^{+}$is nonincreasing and twice differentiable function satisfying for any $t \geq 0$,

$$
\begin{aligned}
k(0) & >0, \\
k^{\prime}(t) & \leq 0 .
\end{aligned}
$$

(A3) There exist a $C^{1}$ function $H: \mathbb{R}^{+} \longrightarrow \mathbb{R}^{+}$, with $H(0)=H^{\prime}(0)=0$, which is linear or is strictly increasing and strictly convex function of class $C^{2}\left(\mathbb{R}^{+}\right)$on $(0, r], r \leq-k^{\prime}(0)$ such that

$$
k^{\prime \prime}(t) \geq \eta(t) H\left(-k^{\prime}(t)\right), \quad \forall t \geq 0,
$$

where $\eta(t)$ is $C^{1}$ nonincreasing continuous function.

Remark 2.1. If assuming further $\lim _{t \rightarrow \infty} k(t)=0$, since $\lim _{t \rightarrow \infty} k(t)=0$ and $\left(-k^{\prime}(t)\right)$ is nonincreasing and nonnegative, we can get

$$
\lim _{t \rightarrow \infty}\left(-k^{\prime}(t)\right)=0 .
$$

Then for some $t_{1} \geq 0$ large,

$$
-k^{\prime}\left(t_{1}\right)=r \Rightarrow-k^{\prime}(t) \leq r, \quad \forall t \geq t_{1} .
$$

Noting that $\left(-k^{\prime}\right)$ is nonincreasing, $-k^{\prime}(0)>0$, and $-k^{\prime}\left(t_{1}\right)>0$, we have $-k^{\prime}\left(t_{1}\right)>0$ for any $t \in\left[0, t_{1}\right]$, and for any $t \in\left[0, t_{1}\right]$,

$$
\begin{aligned}
& 0<-k^{\prime}\left(t_{1}\right) \leq-k^{\prime}(t) \leq-k^{\prime}(0), \\
& 0<\eta\left(t_{1}\right) \leq \eta(t) \leq \eta(0) .
\end{aligned}
$$

Therefore we obtain that there exist two positive constants $a$ and $b$ such that for any $t \in\left[0, t_{1}\right]$,

$$
a \leq \eta(t) H\left(-k^{\prime}(t)\right) \leq b .
$$

Then for any $t \in\left[0, t_{1}\right]$,

$$
k^{\prime \prime}(t) \geq \eta(t) H\left(-k^{\prime}(t)\right) \geq a=\frac{a}{k^{\prime}(0)} k^{\prime}(0) \geq \frac{a}{k^{\prime}(0)} k^{\prime}(t) .
$$

This implies that there exists a constant $d>0$ such that for any $t \in\left[0, t_{1}\right]$,

$$
k^{\prime \prime}(t) \geq-d k^{\prime}(t)
$$

The proof is done.

\section{Main Results}

The well-posedness result is given in [30] proved by using the Faedo-Galerkin method as in [17].
Theorem 1. Assume that (A1) and (A2) hold. Let $\left(u_{0}, u_{1}\right) \in\left(H^{2}(\Omega) \cap V\right) \times V$, and then problem (2) admits a unique solution $u$ satisfying

$$
\begin{array}{r}
u \in L^{\infty}\left(0, T ; H^{2}(\Omega) \cap V\right) \cap W^{1, \infty}(0, T ; V) \\
\cap W^{2, \infty}\left(0, T ; L^{2}(\Omega)\right),
\end{array}
$$

where $V=\left\{v \in H^{1}(\Omega): v=0\right.$ on $\left.\Gamma_{0}\right\}$.

The total energy of the system is defined by

$$
\mathscr{E}(t)=\frac{1}{2}\left\|u_{t}\right\|^{2}+\frac{1}{2}\|\nabla u\|^{2}+\frac{\alpha}{2}\left[k(t)\|u\|_{\Gamma_{1}}^{2}-\int_{\Gamma_{1}} k^{\prime} \mathrm{o} \mathrm{d} \Gamma\right],
$$

where

$$
(k \circ u)(t)=\int_{0}^{t} k(t-s)[u(t)-u(s)]^{2} \mathrm{~d} s .
$$

We can get the following stability result.

Theorem 2. Assume $k$ satisfies (A1)-(A3) and further $\lim _{t \rightarrow \infty} k(t)=0$. Then there exist $\lambda_{1}, \lambda_{2}>0$ such that

$$
\mathscr{E}(t) \leq \lambda_{2} H_{4}^{-1}\left(\lambda_{1} \int_{K^{-1}(r)}^{t} \eta(s) d s\right), \quad \forall t>K^{-1}(r),
$$

where

$$
\begin{aligned}
& H_{4}(t)=\int_{t}^{r} \frac{1}{s H_{0}(s)} \mathrm{d} s, \\
& H_{0}(t)=H^{\prime}(t),
\end{aligned}
$$

and $K(t)=-k^{\prime}(t)$. In particular, if $H(t)=t^{p}$, then for any $t>0$,

$$
\mathscr{E}(t) \leq \begin{cases}c_{1} e^{-c_{2} \int_{0}^{t} \eta(s) \mathrm{d} s}, & \text { if } p=1, \\ c_{3}\left(1+\int_{0}^{t} \eta(s) \mathrm{d} s\right)^{-(1 /(p-1))}, & \text { if } 1<p<2,\end{cases}
$$

where $c_{1}, c_{3}$, and $c_{2} \leq 1$ are positive constants.

Remark 3.1. From (23), the energy result holds for $H(t)=$ $t^{p}$ with the full admissible range $p \in[1,2)$ instead of $p \in[1,(3 / 2))$. If the viscoelastic term is as internal feedback, Lasiecka and Wang [10] provided the proof for optimal decay rates of second-order systems in the full admissible range $[1,2)$.

At last, we show two examples to illustrate explicit formulas for the decay rates of the energy, which can be found in the studies of Mustafa and Mustafa [14, 15].

Example 1. Take $k^{\prime}(t)=-e^{-t^{q}}$ with $0<q<1$, we get $k^{\prime \prime}(t)=H\left(-k^{\prime}(t)\right)$, where $H(t)=\left((q t) /\left([\ln (1 / t)]^{(1 / q)-1}\right)\right)$. Since 


$$
\begin{aligned}
H^{\prime}(t) & =\frac{(1-q)+q \ln (1 / t)}{[\ln (1 / t)]^{(1 / q)}}, \\
H^{\prime \prime}(t) & =\frac{(1-q)[\ln (1 / t)+(1 / q)]}{[\ln (1 / t)]^{((1 / q)+1)}},
\end{aligned}
$$

we can deduce that the function $H$ satisfies (A3) on $(0, r]$ for any $0<r<1$. Then,

$$
\mathscr{E}(t) \leq c_{1} e^{-c_{2} t^{q}}
$$

Example 2. Consider $k^{\prime}(t)=\left(-1 /\left((t+e)[\ln (t+e)]^{p}\right)\right)$ with $p>1$, we $k^{\prime \prime}(t)=\left([\ln (t+e)+p] /\left((t+e)^{2}[\ln (t+e)]^{p+1}\right)\right)$. Clearly,

$$
k^{\prime \prime}(t)=\frac{[\ln (t+e)+p]}{(t+e) \ln (t+e)}\left[-k^{\prime}(t)\right] .
$$

By part 1 of (23), we get

$$
\begin{aligned}
\mathscr{E}(t) & \leq c_{1} \exp \left(-c_{2} \int_{0}^{t} \frac{[\ln (t+e)+p]}{(t+e) \ln (t+e)} \mathrm{d} s\right) \\
& =\frac{c_{1}}{\left[(t+e)(\ln (t+e))^{p}\right]^{c_{2}}} .
\end{aligned}
$$

As $c_{2} \leq 1$, this is slower rate than $\left[-k^{\prime}(t)\right]$. In addition,

$$
k^{\prime \prime}(t)=\frac{[\ln (t+e)+p]}{(t+e)^{(1-(1 / p))}}\left(-k^{\prime}(t)\right)^{(1+(1 / p))} .
$$

From part 2 of (23), we infer that for large $t$

$$
\mathscr{E}(t) c_{3}\left(1+\int_{0}^{t} \frac{\ln (t+e)+p}{(t+e)^{1-(1 / p)}} \mathrm{d} s\right)^{-p} \leq \frac{c_{3}}{(t+e)[\ln (t+e)]^{p}}
$$

which is the same rate as $\left[-k^{\prime}(t)\right]$.

\section{Proof of Main Result}

To prove Theorem 2, we need the following lemmas.

\subsection{Technical Lemmas}

Lemma 1. The total energy functional $E(t)$ satisfies for any $t \geq 0$,

$$
\mathscr{E}^{\prime}(t) \leq-\frac{\alpha}{2}\left(\left\|u_{t}\right\|_{\Gamma_{1}}^{2}+\int_{\Gamma_{1}} k^{\prime \prime \circ} \mathrm{ud} \Gamma\right) \leq 0 .
$$

Proof. See [30].

As in [31], for $0<\delta<1$, we introduce

$$
\begin{aligned}
C_{\delta} & =\int_{0}^{\infty} \frac{\left[k^{\prime}(s)\right]^{2}}{k^{\prime \prime}(s)-\delta k^{\prime}(s)} \mathrm{d} s, \\
h(t) & =k^{\prime \prime}(s)-\delta k^{\prime}(s) .
\end{aligned}
$$

Lemma 2. Define the functional $\Phi(t)$ by

$$
\Phi(t):=\int_{\Omega}[2 m \cdot \nabla u+(n-1) u] u_{t} \mathrm{~d} x
$$

Then we can get for any $t \geq t_{1}$,

$$
\Phi^{\prime}(t) \leq-\left\|u_{t}\right\|^{2}-\frac{1}{2}\|\nabla u\|^{2}+c\left\|u_{t}\right\|_{\Gamma_{1}}^{2}+C_{\delta} \int_{\Gamma_{1}} h \circ u \mathrm{~d} \Gamma .
$$

Proof. From the same arguments as in the study of Mustafa [30], we can obtain

$$
\begin{aligned}
\Phi^{\prime}(t) \leq & -\left\|u_{t}\right\|^{2}-\|\nabla u\|^{2}-\delta_{0}\|\nabla u\|_{\Gamma_{1}}^{2}+\int_{\Gamma_{1}}(m \cdot \nu)\left|u_{t}\right|^{2} \mathrm{~d} \Gamma \\
& +\int_{\Gamma_{1}}(2 m \cdot \nabla u) \frac{\partial u}{\partial \nu} \mathrm{d} \Gamma+(n-1) \int_{\Gamma_{1}} u \frac{\partial u}{\partial \nu} \mathrm{d} \Gamma .
\end{aligned}
$$

It follows from Young's inequality that for any $\varepsilon>0$,

$$
\begin{aligned}
& \int_{\Gamma_{1}}(2 m \cdot \nabla u) \frac{\partial u}{\partial \nu} \mathrm{d} \Gamma+(n-1) \int_{\Gamma_{1}} u \frac{\partial u}{\partial \nu} \mathrm{d} \Gamma \\
& \leq \delta_{0}\|\nabla u\|_{\Gamma_{1}}^{2}+\varepsilon\|u\|_{\Gamma_{1}}^{2}+c\left\|\frac{\partial u}{\partial \nu}\right\|_{\Gamma_{1}}^{2} .
\end{aligned}
$$

Recalling $\quad k^{\prime} * u=\left(-k^{\prime} \odot u\right)+[k(t)-k(0)] u$, where $k \odot u=\int_{0}^{t} k(t-s)(u(t)-u(s)) \mathrm{d} s$; then we have from (8),

$$
\frac{\partial u}{\partial v}(t)=-\alpha\left[u_{t}(t)+k(t) u(t)+\left(-k^{\prime} \odot u\right)(t)\right] .
$$

By using Young's inequality, we obtain 


$$
\left\|\frac{\partial u}{\partial v}(t)\right\|_{\Gamma_{1}}^{2} \leq 4 \alpha^{2}\left[\left\|u_{t}\right\|_{\Gamma_{1}}^{2}+k^{2}(t)\|u\|_{\Gamma_{1}}^{2}+\int_{\Gamma_{1}}\left(-k^{\prime} \odot u\right)^{2} \mathrm{~d} \Gamma\right] . \quad \text { Hölder's inequality implies }
$$

$$
\begin{aligned}
\left(-k^{\prime} \odot u\right)^{2} & =\left(\int_{0}^{t}\left(-k^{\prime}(t-s)\right)(u(t)-u(s)) \mathrm{d} s\right)^{2} \\
& =\left(\int_{0}^{t} \frac{-k^{\prime}(t-s)}{\sqrt{k^{\prime \prime}(t-s)-\delta k^{\prime}(t-s)}} \sqrt{k^{\prime \prime}(t-s)-\delta k^{\prime}(t-s)}(u(t)-u(s)) \mathrm{d} s\right)^{2} \\
& \leq\left(\int_{0}^{t} \frac{\left[k^{\prime}(s)\right]^{2}}{k^{\prime \prime}(s)-\delta k^{\prime}(s)} \mathrm{d} s\right) \int_{0}^{t}\left(k^{\prime \prime}(t-s)-\delta k^{\prime}(t-s)\right)(u(t)-u(s))^{2} \mathrm{~d} s \\
& \leq C_{\delta}(h \circ u),
\end{aligned}
$$

which, together with (37), gives us that

$$
\left\|\frac{\partial u}{\partial \nu}(t)\right\|_{\Gamma_{1}}^{2} \leq 4 \alpha^{2}\left[\left\|u_{t}\right\|_{\Gamma_{1}}^{2}+k^{2}(t)\|u\|_{\Gamma_{1}}^{2}+C_{\delta} \int_{\Gamma_{1}}(h \circ u) \mathrm{d} \Gamma\right] .
$$

Inserting (39) into (35), we obtain for any $\varepsilon>0$,

$$
\begin{aligned}
& \int_{\Gamma_{1}}(2 m \cdot \nabla u) \frac{\partial u}{\partial \nu} \mathrm{d} \Gamma+(n-1) \int_{\Gamma_{1}} u \frac{\partial u}{\partial \nu} \mathrm{d} \Gamma \\
& \leq \delta_{0}\|\nabla u\|_{\Gamma_{1}}^{2}+\left(\varepsilon+4 \alpha^{2} k^{2}(t)\right)\|u\|_{\Gamma_{1}}^{2} \\
& +4 \alpha^{2} c\left\|u_{t}\right\|_{\Gamma_{1}}^{2}+C_{\delta} \int_{\Gamma_{1}}(h \circ u) \mathrm{d} \Gamma .
\end{aligned}
$$

Noting that

$$
\|u\|_{\Gamma_{1}}^{2} \leq c\|\nabla u\|^{2}
$$

using $\lim _{t \rightarrow \infty} k(t)=0$ and taking $\varepsilon>0$ small enough, we can get (33) from (34) and (40). The proof is done.

To get the optimal energy decay, we need the following estimate.
Lemma 3. The functional $\Psi(t)$ is defined by

$$
\Psi(t):=\int_{0}^{t} k(t-s)\|u(s)\|_{\Gamma_{1}}^{2} \mathrm{~d} s,
$$

which satisfies for any $t>0$,

$$
\Psi^{\prime}(t) \leq \frac{1}{2} \int_{\Gamma_{1}} k^{\prime}{ }^{\circ} \mathrm{ud} \Gamma+3 k(0)\|u(t)\|_{\Gamma_{1}}^{2} .
$$

Proof. Differentiating $\Psi(t)$ with respect to $t$, we get

$$
\begin{aligned}
\Psi^{\prime}(t)= & k_{2}(0)\|u(t)\|_{\Gamma_{1}}^{2}+\int_{0}^{t} k_{2}^{\prime}(t-s)\|u(s)\|_{\Gamma_{1}}^{2} \mathrm{~d} s \\
= & \int_{0}^{t} k^{\prime}(t-s) \int_{\Gamma_{1}}[u(s)-u(t)]^{2} \mathrm{~d} \Gamma \mathrm{d} s+k(t)\|u(t)\|_{\Gamma_{2}}^{2} \\
& +2 \int_{\Gamma_{1}} u(t) \int_{0}^{t} k^{\prime}(t-s)[u(s)-u(t)] \mathrm{d} s \mathrm{~d} \Gamma .
\end{aligned}
$$

In view of Young's and Hölder's inequalities, we obtain

$$
\begin{aligned}
& 2 \int_{\Gamma_{1}} u(t) \int_{0}^{t} k^{\prime}(t-s)\left[u(s)-u(t) \sqrt{a^{2}+b^{2}}\right] \mathrm{d} s \mathrm{~d} \Gamma \\
& \quad \leq 2 k(0) \int_{\Gamma_{1}} u^{2}(t) \mathrm{d} \Gamma+\frac{1}{2 k(0)} \int_{\Gamma_{1}}\left(\int_{0}^{t} \sqrt{-k^{\prime}(t-s)} \sqrt{-k^{\prime}(t-s)}[u(s)-u(t)] \mathrm{d} s\right)^{2} \mathrm{~d} \Gamma \\
& \quad \leq 2 k(0)\|u(t)\|_{\Gamma_{1}}^{2}+\frac{\int_{0}^{t} k^{\prime}(s) \mathrm{d} s}{2 k(0)} \int_{0}^{t} k^{\prime}(t-s)\|u(s)-u(t)\|_{\Gamma_{1}}^{2} \mathrm{~d} s .
\end{aligned}
$$


Then we can get (43) following from the fact

$$
\begin{array}{r}
k(t) \leq k(0), \\
\frac{\int_{0}^{t} k^{\prime}(s) \mathrm{d} s}{2 k(0)} \geq-\frac{1}{2} .
\end{array}
$$

The proof is complete.

\subsection{Proof of Theorem 2}

Proof. Define the functional $L(t)$ by

$$
L(t):=N \mathscr{E}(t)+\Phi(t)
$$

where $N>0$ is a constant that will be taken later. Clearly we can take $N$ a large value to get

$$
L(t) \sim \mathscr{E}(t) .
$$

Recalling $k^{\prime \prime}=\delta k^{\prime}+h$, combining (30) and (33), we conclude that for any $t>t_{1}$,

$$
\begin{gathered}
L^{\prime}(t) \leq-\left(\frac{\alpha}{2} N-c\right)\left\|u_{t}\right\|_{\Gamma_{1}}^{2}-\left\|u_{t}\right\|^{2}-\frac{1}{2}\|\nabla u\|^{2} \\
\cdot-\frac{\alpha}{2} N \delta \int_{\Gamma_{1}} k^{\prime}{ }^{\circ} \mathrm{ud} \Gamma-\left(\frac{\alpha}{2} N-c C_{\delta}\right) \int_{\Gamma_{1}} h^{\circ} \mathrm{ud} \Gamma .
\end{gathered}
$$

Noting $-k^{\prime}>0$ and $k^{\prime \prime}>0$, for each $s \in[0, \infty)$, we shall see below,

$$
\begin{aligned}
\lim _{\delta \rightarrow 0} \frac{\delta\left[k^{\prime}(s)\right]^{2}}{k^{\prime \prime}(s)-\delta k^{\prime}(s)} \mathrm{d} s & =0, \\
\frac{\delta\left[k^{\prime}(s)\right]^{2}}{k^{\prime \prime}(s)-\delta k^{\prime}(s)} & <-k^{\prime}(s) .
\end{aligned}
$$

It follows from Lebesgue dominated convergence theorem that

$$
\lim _{\delta \rightarrow 0} \delta C_{\delta}=\lim _{\delta \rightarrow 0} l \int_{0}^{\infty} \frac{\delta\left[k^{\prime}(s)\right]^{2}}{k^{\prime \prime}(s)-\delta k^{\prime}(s)} \mathrm{d} s=0 .
$$

Therefore there exist $0<\gamma<1$ such that if $\delta<\gamma$, then

$$
\delta C_{\delta}<\frac{1}{4 c} \text {. }
$$

And then we choose $N$ a larger value that

$$
\frac{\alpha}{2} N-c>4 k(0)
$$

and take $\delta>0$ satisfying

$$
\delta=\frac{1}{2 \alpha N}<\gamma
$$

This implies

$$
\frac{\alpha}{2} N-c C_{\delta}>0
$$
$t_{1}>0$,

$$
\begin{aligned}
L^{\prime}(t) \leq & -\beta\left(\left\|u_{t}\right\|^{2}+\|\nabla u\|^{2}\right)-4 k(0)\left\|u_{t}\right\|_{\Gamma_{1}}^{2} \\
& -\frac{1}{4} \int_{\Gamma_{1}} k^{\prime}{ }^{\circ} \mathrm{ud} \Gamma, \quad \forall t \geq t_{1} .
\end{aligned}
$$

By (17) and (30), we get

$$
\int_{0}^{t_{1}}\left(-k^{\prime}(s)\right) \int_{\Gamma_{1}}[u(t)-u(t-s)]^{2} \mathrm{~d} \Gamma \mathrm{d} s
$$

$$
\leq \frac{1}{d} \int_{0}^{t_{1}} k^{\prime \prime}(s) \int_{\Gamma_{1}}[u(t)-u(t-s)]^{2} \mathrm{~d} \Gamma \mathrm{d} s \leq-c \mathscr{E}^{\prime}(t) .
$$

Then from (56), we infer that there exists a constant $\chi>0$ such that

$$
\begin{aligned}
L^{\prime}(t) & \leq-\chi \mathscr{E}(t)-c \int_{0}^{t} k^{\prime}(s) \int_{\Gamma_{1}}[u(t)-u(t-s)]^{2} \mathrm{~d} \Gamma \mathrm{d} s \\
& \leq-\chi \mathscr{E}(t)-c \mathscr{E}^{\prime}(t)-c \int_{t_{1}}^{t} k^{\prime}(s) \int_{\Gamma_{1}}[u(t)-u(t-s)]^{2} \mathrm{~d} \Gamma \mathrm{d} s .
\end{aligned}
$$

Denoting $F(t):=L(t)+c \mathscr{E}(t) \sim E(t)$, and using (58), we know that

$$
F^{\prime}(t) \leq-\chi \mathscr{E}(t)-c \int_{t_{1}}^{t} k_{2}^{\prime}(s) \int_{\Gamma_{1}}[u(t)-u(t-s)]^{2} \mathrm{~d} \Gamma \mathrm{d} s .
$$

In the sequel, we consider two cases.

Case 1. The particular case $H(t)=t^{p}$.

(I) $p=1$.

Multiplying (59) by $\eta(t)$, and using (19) and (A2)-(A3), we have

$$
\eta(t) F^{\prime}(t) \leq-\chi \eta(t) \mathscr{E}(t)-c \mathscr{E}^{\prime}(t), \quad \forall t \geq t_{1}
$$

Since $\eta(t)$ is a nonincreasing continuous function and $\eta^{\prime}(t) \leq 0$ for a.e. $t$, then

$$
\begin{aligned}
(\eta F+c \mathscr{E})^{\prime}(t) & \leq \eta(t) F^{\prime}(t)+c \mathscr{E}^{\prime}(t) \\
& \leq-m \eta(t) \mathscr{E}(t), \quad \text { a.e. } t \geq t_{1} .
\end{aligned}
$$

In view of $\eta F+c \mathscr{E} \sim \mathscr{E}$, we obtain that there exist two positive constants $c_{1}, c_{2}>0$,

$$
\mathscr{E}(t) \leq c_{1} e^{-c_{2} \int_{t_{1}}^{t} \eta(s) \mathrm{d} s}
$$

(II) $1<p<2$

Define $\mathscr{G}(t)$ by

$$
\mathscr{G}(t)=L(t)+\Psi(t) .
$$

It follows from (43) and (56) that $\mathscr{E}(t) \geq 0$, and for any $t \geq t_{1}$, 


$$
\mathscr{G}^{\prime}(t) \leq-\beta\left(\left\|u_{t}\right\|^{2}+\|\nabla u\|^{2}\right)-k(0)\left\|u_{t}\right\|_{\Gamma_{1}}^{2}+\frac{1}{4} \int_{\Gamma_{1}} k^{\prime}{ }^{\circ} \mathrm{ud} \Gamma .
$$

Then there exists a certain constant $\beta_{1}>0$,

$$
\mathscr{G}^{\prime}(t) \leq-\beta_{1} \mathscr{E}(t), \quad \forall t \geq t_{1} .
$$

This gives us

$$
\beta_{1} \int_{t_{1}}^{t} E(s) \mathrm{d} s \leq \mathscr{G}\left(t_{1}\right)-\mathscr{G}(t) \leq \mathscr{G}\left(t_{1}\right) .
$$

Hence

$$
\int_{0}^{\infty} \mathscr{E}(s) \mathrm{d} s<\infty
$$

Define

$$
I(t)=\int_{0}^{t} \int_{\Gamma_{1}}[u(t)-u(t-s)]^{2} \mathrm{~d} \Gamma \mathrm{d} s
$$

we know that

$$
I(t) \leq c \int_{0}^{t} \mathscr{E}(s) \mathrm{d} s
$$

Without loss of generality assuming $t_{1}$ so large that $I\left(t_{1}\right)>0$, then

$$
0<I\left(t_{1}\right) \leq I(t)<\infty, \quad \forall t \geq t_{1} .
$$

Using Jensen's inequality and by (30) and (A2)-(A3), we can derive from (56) that for some constant $q>0$,

$$
\begin{aligned}
L^{\prime}(t) & \leq-q \mathscr{E}(t)+\frac{c I(t)}{I(t)} \int_{\Gamma_{1}}\left[\left(-k^{\prime}\right)^{p \cdot(1 / p)} \circ u\right] \mathrm{d} \Gamma \\
& \leq-q \mathscr{E}(t)+c I(t)\left[\frac{1}{I(t)} \int_{\Gamma_{1}}\left(-k^{\prime}\right)^{p} \circ u \mathrm{~d} \Gamma\right]^{(1 / p)} \\
& \leq-q \mathscr{E}(t)+c I^{1-(1 / p)}(t)\left[\int_{\Gamma_{1}} \frac{k^{\prime \prime}}{\eta} u \mathrm{~d} \Gamma\right]^{(1 / p)} \\
& \leq-q \mathscr{E}(t)+\frac{c}{[\eta(t)]^{(1 / p)}}\left[\int_{\Gamma_{1}} k^{\prime \prime} \circ u \mathrm{~d} \Gamma\right]^{(1 / p)} \\
& \leq-q \mathscr{E}(t)+\frac{c}{[\eta(t)]^{(1 / p)}}\left[-\mathscr{E}^{\prime}(t)\right]^{(1 / p)}
\end{aligned}
$$

We multiply (71) by $\mathscr{E}^{p^{-1}}(t)$ and use (19) to deduce

$$
\begin{aligned}
\left(L \mathscr{E}^{p-1}\right)^{\prime}(t) \leq & L^{\prime}(t) \mathscr{E}^{p^{-1}}(t) \leq-q \mathscr{E}^{p}(t) \\
& +c\left[-\frac{\mathscr{E}^{\prime}(t)}{\eta(t)}\right]^{(1 / p)} \mathscr{E}^{p-1}(t) .
\end{aligned}
$$

By Young's inequality, we have for any $\varepsilon_{1}>0$,

$$
\left(L \mathscr{E}^{p-1}\right)^{\prime}(t) \leq-q \mathscr{E}^{p}(t)+\varepsilon_{1} \mathscr{E}^{p}(t)+\frac{c}{\varepsilon_{1}}\left[-\frac{\mathscr{E}^{\prime}(t)}{\eta(t)}\right] .
$$

Taking $\varepsilon_{1}<(1 / 2) q$, we conclude

$$
\left(L \mathscr{E}^{p-1}\right)^{\prime}(t) \leq-\frac{q}{2} \mathscr{E}^{p}(t)-c \frac{\mathscr{E}^{\prime}(t)}{\eta(t)} .
$$

Define $F(t)=\eta L \mathscr{E}^{p-1}+c \mathscr{E} \sim \mathscr{E}$. Multiplying (74) by $\eta(t)$, we have

$$
F^{\prime}(t) \leq-\frac{q}{2} \eta(t) \mathscr{E}^{p}(t)
$$

Then there exists a certain constant $q_{0}>0$ such that

$$
F^{\prime}(t) \leq-q_{0} \eta(t) F^{p}(t)
$$

from which we obtain

$$
\mathscr{E}(t) \leq c_{3}\left(1+\int_{0}^{t} \eta(s) \mathrm{d} s\right)^{-(1 /(p-1))},
$$

where $c_{3}$ is a positive constant.

Combining (I) and (II) and using the boundedness of $\eta(t)$ and $\mathscr{E}(t)$, we can get $(23)$.

Case 2. The general case.

Define

$$
I(t):=q \int_{t_{1}}^{t} \int_{\Gamma_{1}}[u(t)-u(t-s)]^{2} \mathrm{~d} \Gamma \mathrm{d} s .
$$

In view of (67), we can take $0<q<1$ such that

$$
I(t)<1, \quad \forall t \geq t_{1} .
$$

Without loss of generality, we assume that $I(t)>0$ for all $t \geq t_{1}$. On the other hand, we define

$$
\pi(t):=\int_{t_{1}}^{t} k^{\prime \prime}(s) \int_{\Gamma_{1}}[u(t)-u(t-s)]^{2} \mathrm{~d} \Gamma \mathrm{d} s .
$$

From (30), we can easily get $\pi(t) \leq-c \mathrm{E}^{\prime}(t)$. As $H(t)$ is strictly convex on $(0, r]$ and $H(0)=0$, we see that 
$H(\lambda x) \leq \lambda H(x), \quad i=1,2,0 \leq \lambda \leq 1, x \in(0, r]$.

It follows from Jensen's inequality and (11) and (79) that

$$
\begin{aligned}
\pi_{1}(t) & =\frac{1}{q I(t)} \int_{t_{1}}^{t} I(t)\left(k^{\prime \prime}(s)\right) q \int_{\Gamma_{1}}[u(t)-u(t-s)]^{2} \mathrm{~d} \Gamma \mathrm{d} s \\
& \geq \frac{1}{q I(t)} \int_{t_{1}}^{t} I(t) \eta(s) H\left(-k^{\prime}(s)\right) q \int_{\Gamma_{1}}[u(t)-u(t-s)]^{2} \mathrm{~d} \Gamma \mathrm{d} s \\
& \geq \frac{\eta(t)}{q I(t)} \int_{t_{1}}^{t} H\left(I(t)\left(-k^{\prime}(s)\right)\right) q \int_{\Gamma_{1}}[u(t)-u(t-s)]^{2} \mathrm{~d} \Gamma \mathrm{d} s \\
& \geq \frac{\eta(t)}{q} H\left(\frac{1}{I(t)} \int_{t_{1}}^{t} I(t)\left(-k^{\prime}(s)\right) q \int_{\Gamma_{1}}[u(t)-u(t-s)]^{2} \mathrm{~d} \Gamma \mathrm{d} s\right) \\
& =\frac{\eta(t)}{q} H\left(q \int_{t_{1}}^{t}\left(-k^{\prime}\right)(s) \int_{\Gamma_{1}}[u(t)-u(t-s)]^{2} \mathrm{~d} \Gamma \mathrm{d} s\right) \\
& =\frac{\eta(t)}{q} \bar{H}\left(q \int_{t_{1}}^{t}\left(-k^{\prime}(s)\right) \int_{\Gamma_{1}}[u(t)-u(t-s)]^{2} \mathrm{~d} \Gamma \mathrm{d} s\right),
\end{aligned}
$$

where $\bar{H}$, which is strictly convex and increasing function on $(0, \infty)$ of class $C^{2}$, is called the extension of $H$. We infer from (82) that

$$
\int_{t_{1}}^{t}\left(-k^{\prime}(s)\right) \int_{\Gamma_{1}}[u(t)-u(t-s)]^{2} \mathrm{~d} \Gamma \mathrm{d} s \leq \frac{1}{q} \bar{H}^{-1}\left(\frac{q \pi(t)}{\eta(t)}\right) .
$$

Then we can get from (59) that for any $t \geq t_{1}$,

$$
F^{\prime}(t) \leq-\chi \mathscr{E}(t)+c \bar{H}^{-1}\left(\frac{q \pi(t)}{\eta(t)}\right) .
$$

$$
\begin{aligned}
\mathscr{K}_{1}^{\prime}(t) & =r_{0} \frac{\mathscr{E}^{\prime}(t)}{\mathscr{E}(0)} H_{0}^{\prime}\left(r_{0} \frac{\mathscr{E}(t)}{\mathscr{E}(0)}\right) F(t)+H_{0}\left(r_{0} \frac{\mathscr{E}(t)}{\mathscr{E}(0)}\right) F^{\prime}(t)+\mathscr{E}^{\prime}(t) \\
& \leq-m \mathscr{E}(t) H_{0}\left(r_{0} \frac{\mathscr{E}(t)}{\mathscr{E}(0)}\right)+c H_{0}\left(r_{0} \frac{\mathscr{E}(t)}{\mathscr{E}(0)}\right) \bar{H}^{-1}\left(\frac{q \pi(t)}{\eta(t)}\right) .
\end{aligned}
$$

We denote by $\bar{H}^{*}$ the conjugate function of the convex function $\bar{H}$ (see Arnold [33]), and then

$$
\bar{H}^{*}(s)=s\left(\bar{H}^{\prime}\right)^{-1}(s)-\bar{H}\left[\left(\bar{H}^{\prime}\right)^{-1}(s)\right]
$$

\section{Denote}

$$
H_{0}(t)=\bar{H}^{\prime}(t)
$$

For $r_{0}<r$, we define $\mathscr{K}_{1}(t)$ by

$$
\mathscr{K}_{1}(t)=H_{0}\left(r_{0} \frac{\mathscr{E}(t)}{\mathscr{E}(0)}\right) F(t)+\mathscr{E}(t) \sim \mathscr{E}(t) .
$$

Since $E^{\prime}(t) \leq 0, \bar{H}^{\prime}>0$, and $\bar{H}^{\prime \prime}>0$, we get from (84) that 
Taking $A=\bar{H}_{0}^{\prime}\left(r_{0}(\mathrm{E}(t) / \mathrm{E}(0))\right)$ and $B=\bar{H}^{-1}((q \pi(t))$ $/ \eta(t))$, and using $\bar{H}^{*}(s) \leq s\left(\bar{H}^{\prime}\right)^{-1}(s)$ and (87), we have

$$
\begin{aligned}
\mathscr{K}_{1}^{\prime}(t) & \leq-\chi \mathscr{E}(t) H_{0}\left(r_{0} \frac{\mathscr{E}(t)}{\mathscr{E}(0)}\right)+c \bar{H}^{*}\left(H_{0}\left(r_{0} \frac{\mathscr{E}(t)}{\mathscr{E}(0)}\right)\right)+c \frac{q \pi(t)}{\eta(t)} \\
& \leq-\chi \mathscr{E}(t) H_{0}\left(r_{0} \frac{\mathscr{E}(t)}{\mathscr{E}(0)}\right)+c H_{0}\left(r_{0} \frac{\mathscr{E}(t)}{\mathscr{E}(0)}\right)\left(\bar{H}^{\prime}\right)^{-1}\left(H_{0}\left(r_{0} \frac{\mathscr{E}(t)}{\mathscr{E}(0)}\right)\right)+c \frac{q \pi(t)}{\eta(t)} \\
& \leq-\chi \mathscr{E}(t) H_{0}\left(r_{0} \frac{\mathscr{E}(t)}{\mathscr{E}(0)}\right)+c H_{0}\left(r_{0} \frac{\mathscr{E}(t)}{\mathscr{E}(0)}\right)\left(\bar{H}^{\prime}\right)^{-1}\left(\bar{H}^{\prime}\left(r_{0} \frac{\mathscr{E}(t)}{\mathscr{E}(0)}\right)\right)+c \frac{q \pi(t)}{\eta(t)} \\
& \leq-\left(\chi \mathscr{E}(0)-c r_{0}\right) \frac{\mathscr{E}(t)}{\mathscr{E}(0)} H_{0}\left(r_{0} \frac{\mathscr{E}(t)}{\mathscr{E}(0)}\right)+c q \frac{\pi(t)}{\eta_{1}(t)}
\end{aligned}
$$

We multiply (90) by $\eta(t)$ to arrive at

$$
\begin{aligned}
\eta(t) \mathscr{K}_{1}^{\prime}(t) \leq-\left(\chi^{\mathscr{E}}(0)-c r_{0}\right) \eta(t) \frac{\mathscr{E}(t)}{\mathscr{E}(0)} H_{0}\left(r_{0} \frac{\mathscr{E}(t)}{\mathscr{E}(0)}\right)+c q \pi(t) \\
\leq-\left(\chi^{\mathscr{E}(0)}-c r_{0}\right) \eta(t) \frac{\mathscr{E}(t)}{\mathscr{E}(0)} H_{0}\left(r_{0} \frac{\mathscr{E}(t)}{\mathscr{E}(0)}\right)-c^{\mathscr{E}^{\prime}}(t) . \\
\quad H_{0}\left(r_{0} \frac{\mathscr{E}(t)}{\mathscr{E}(0)}\right)=\bar{H}^{\prime}\left(r_{0} \frac{\mathscr{E}(t)}{\mathscr{E}(0)}\right)=H^{\prime}\left(r_{0} \frac{\mathscr{E}(t)}{\mathscr{E}(0)}\right) .
\end{aligned}
$$

The functional $\mathscr{K}_{2}(t)$ is defined by

$$
\mathscr{K}_{2}(t)=\eta(t) \mathscr{K}_{1}(t)+c \mathscr{E}(t) .
$$

Then we can easily obtain that there exist constants $\beta_{5}>0$ and $\beta_{6}>0$ such that

$$
\beta_{5} \mathscr{K}_{2}(t) \leq \mathrm{E}(t) \leq \beta_{6} \mathscr{K}_{2}(t) .
$$

Choosing a suitable $r_{0}>0$, and defining $H_{3}(t)=t H_{0}\left(r_{0} t\right)$, from (91), we infer that for a constant $\gamma>0$,

$$
\mathscr{K}_{2}^{\prime}(t) \leq-\gamma \eta(t) \frac{\mathscr{E}(t)}{\mathscr{E}(0)} H_{0}\left(r_{0} \frac{\mathscr{E}(t)}{\mathscr{E}(0)}\right):=-\gamma \eta(t) H_{3}\left(\frac{\mathscr{E}(t)}{\mathscr{E}(0)}\right) .
$$

Using (93), we have

$$
R(t):=\frac{\beta_{5} \mathscr{K}_{2}(t)}{\mathscr{E}(0)} \sim \mathscr{E}(t) .
$$

Since $H_{3}^{\prime}(t)=H_{0}\left(r_{0} t\right)+r_{0} t H_{0}^{\prime}\left(r_{0} t\right)$, then, noting the strict convexity of $H_{0}$ on $(0, r]$, we know $H_{3}^{\prime}(t), H_{3}(t)>0$ on $(0,1]$. By (94), we conclude that there exists $\gamma_{1}>0$ such that for any $t \geq t_{1}$,

$$
R^{\prime}(t) \leq-\gamma_{1} \eta(t) H_{3}(R(t)) .
$$

Integrating (97) over $\left(t_{1}, t\right)$, we see that

It follows from $0 \leq r_{0}(\mathscr{E}(t) / \mathscr{E}(0))<r$ that for any $t>0$,

$$
\int_{t_{1}}^{t} \frac{-R^{\prime}(s)}{H_{3}(R(s))} \mathrm{d} s \geq \gamma_{1} \int_{t_{1}}^{t} \eta(s) \mathrm{d} s \Rightarrow \int_{r_{0} R(t)}^{r_{0} R\left(t_{1}\right)} \frac{1}{s H_{0}(s)} \mathrm{d} s \geq \gamma_{1} \int_{t_{1}}^{t} \eta(s) \mathrm{d} s .
$$

Define

$$
H_{4}(t)=\int_{t}^{r} \frac{1}{s H_{0}(s)} \mathrm{d} s
$$

It is to verify that $\mathrm{H}_{4}$ is strictly decreasing on $(0, r]$ and $\lim _{t \longrightarrow 0} H_{4}(t)=+\infty$. It follows that

$$
R(t) \leq \frac{1}{r_{0}} H_{4}^{-1}\left(\zeta_{1} \int_{t_{1}}^{t} \eta(s) \mathrm{d} s\right) .
$$


Combining (96) and (100), we can obtain (21). This finishes the proof of Theorem 2

\section{Data Availability}

No data were used during this study.

\section{Conflicts of Interest}

The author declares that there are no conflicts of interest regarding the publication of this paper.

\section{Acknowledgments}

This work was supported by the Fundamental Research Funds for the Central Universities (no. JBK1809025).

\section{References}

[1] J. Prüss, "Evolutionary integral equations and applications," in Monographs in Mathematics, vol. 87, Birkhäuser Verlag, Basel, Switzerland, 1993.

[2] A. Eden, C. Foias, B. Nicolaenko, and R. Temam, "Exponential attractors for dissipative evolution equations," in RAM: Research in Applied Mathematics, vol. 37, Masson, Paris, France, 1994.

[3] B. Feng, M. A. Jorge Silva, and A. H. Caixeta, "Long-time behavior for a class of semi-linear viscoelastic Kirchhoff beams/plates," Applied Mathematics \& Optimization, vol. 82, no. 2, pp. 657-686, 2020.

[4] S. A. Messaoudi, "General decay of solutions of a viscoelastic equation," Journal of Mathematical Analysis and Applications, vol. 341, no. 2, pp. 1457-1467, 2008.

[5] S. A. Messaoudi, "General decay of the solution energy in a viscoelastic equation with a nonlinear source," Nonlinear Analysis: Theory, Methods \& Applications, vol. 69, no. 8, pp. 2589-2598, 2008.

[6] I. Lasiecka, S. A. Messaoudi, and M. I. Mustafa, "Note on intrinsic decay rates for abstract wave equations with memory," Journal of Mathematical Physics, vol. 54, p. 31504, 2013.

[7] F. Alabau-Boussouira and P. Cannarsa, "A general method for proving sharp energy decay rates for memory-dissipative evolution equations," Comptes Rendus Mathematique, vol. 347, no. 15-16, pp. 867-872, 2009.

[8] M. M. Cavalcanti, V. N. Domingos Cavalcanti, I. Lasiecka, and F. A. Nascimento, "Intrinsic decay rate estimates for the wave equation with competing viscoelastic and frictional dissipative effects," Discrete \& Continuous Dynamical System _ B, vol. 19, pp. 1987-2012, 2014.

[9] M. M. Cavalcanti, A. D. D. Cavalcanti, I. Lasiecka, and $\mathrm{X}$. Wang, "Existence and sharp decay rate estimates for a von Karman system with long memory," Nonlinear Analysis: Real World Applications, vol. 22, pp. 289-306, 2015.

[10] I. Lasiecka and X. Wang, "Intrinsic decay rate estimates for semilinear abstract second order equations with memory," in New Prospects in Direct, Inverse and Control Problems for Evolution Equations, vol. 10, pp. 271-303, Springer INdAM Series, Cham, Switzerland, 2014.

[11] M. I. Mustafa and S. A. Messaoudi, "General stability result for viscoelastic wave equations," Journal of Mathematical Physics, vol. 53, p. 53702, 2012.

[12] T.-J. Xiao and J. Liang, "Coupled second order semilinear evolution equations indirectly damped via memory effects,"
Journal of Differential Equations, vol. 254, no. 5, pp. 21282157, 2013.

[13] B. Feng and A. Soufyane, "Optimal decay rates of a nonlinear time-delayed viscoelastic wave equation," International Journal of Differential Equations, vol. 33, pp. 43-65, 2020.

[14] M. I. Mustafa, "Optimal decay rates for the viscoelastic wave equation," Mathematical Methods in the Applied Sciences, vol. 41, no. 1, pp. 192-204, 2018.

[15] M. I. Mustafa, "General decay result for nonlinear viscoelastic equations," Journal of Mathematical Analysis and Applications, vol. 457, no. 1, pp. 134-152, 2018.

[16] M. L. Santos, "Asymptotic behavior of solutions to wave equations with a memory conditions at the boundary," Electron. J. Differ. Equ.vol. 73, p. 11, 2001.

[17] M. L. Santos, J. Ferreira, D. C. Pereira, and C. A. Raposo, "Global existence and stability for wave equation of Kirchhoff type with memory condition at the boundary," Nonlinear Analysis: Theory, Methods \& Applications, vol. 54, no. 5, pp. 959-976, 2003.

[18] J. Y. Park, J. J. Bae, and I. H. Jung, "Uniform decay of solution for wave equation of Kirchhoff type with nonlinear boundary damping and memory term," Nonlinear Analysis: Theory, Methods \& Applications, vol. 50, no. 7, pp. 871-884, 2002.

[19] M. d. L. Santos and F. Junior, "A boundary condition with memory for Kirchhoff plates equations," Applied Mathematics and Computation, vol. 148, no. 2, pp. 475-496, 2004.

[20] M. M. Cavalcanti, V. N. D. Cavalcanti, J. S. P. Filho, and J. A. Soriano, "Existence and uniform decay of solutions of a degenerate equation with nonlinear boundary damping and boundary memory source term," Nonlinear Analysis: Theory, Methods \& Applications, vol. 38, no. 3, pp. 281-294, 1999.

[21] S. A. Messaoudi and A. Soufyane, "General decay of solutions of a wave equation with a boundary control of memory type," Nonlinear Analysis: Real World Applications, vol. 11, no. 4, pp. 2896-2904, 2010.

[22] S. T. Wu, "General decay for a wave equation of Kirchhoff type with a boundary control of memory type," Boundary Value Problems, vol. 2011, p. 15, 2011.

[23] M. M. Cavalcanti and A. Guesmia, "General decay rates of solutions to a nonlinear wave equation with boundary conditions of memory type," Differential Intermediate Equations, vol. 18, pp. 583-600, 2005.

[24] B. Feng, "General decay rates for a viscoelastic wave equation with dynamic boundary conditions and past history," Mediterranean Journal of Mathematics, vol. 15, p. 103, 2018.

[25] B. Feng and A. Soufyane, "New general decay results for a von Karman plate equation with memory-type boundary conditions," Discrete \& Continuous Dynamical Systems - A, vol. 40, no. 3, pp. 1757-1774, 2020.

[26] B. Feng and A. Soufyane, "Existence and decay rates for a coupled Balakrishnan-Taylor viscoelastic system with dynamic boundary conditions," Mathematical Methods in the Applied Sciences, vol. 43, no. 6, pp. 3375-3391, 2020.

[27] B. Feng and A. Soufyane, "Memory-type boundary control of a laminated Timoshenko beam," Mathematics and Mechanics of Solids, vol. 25, no. 8, pp. 1568-1588, 2020.

[28] J. E. Muñoz Rivera and D. Andrade, "Exponential decay of non-linear wave equation with a viscoelastic boundary condition," Mathematical Methods in the Applied Sciences, vol. 23, no. 1, pp. 41-61, 2000.

[29] Q. Zhang, "Global existence and exponential stability for a quasilinear wave equation with memory damping at the boundary," Journal of Optimization Theory and Applications, vol. 139, no. 3, pp. 617-634, 2008. 
[30] M. I. Mustafa, "On the control of the wave equation by memory-type boundary condition," Discrete and Continuous Dynamical Systems Journal, vol. 35, pp. 1179-1192, 2015.

[31] K.-P. Jin, J. Liang, and T.-J. Xiao, "Coupled second order evolution equations with fading memory: optimal energy decay rate," Journal of Differential Equations, vol. 257, no. 5, pp. 1501-1528, 2014

[32] I. Lasiecka and D. Tataru, "Uniform boundary stabilization of semilinear wave equation with nonlinear boundary damping," Differential Intermediate Equations, vol. 8, pp. 507-533, 1993.

[33] V. I. Arnold, Mathematical Methods of Classical Mechanics, Springer-Verlag, New York, NY, USA, 1989. 